\title{
The primary health care performance initiative (PHCPI): issues and challenges for Malaysia as a trailblazer country
}

\begin{abstract}
A strong and robust Primary Health Care system is essential to achieving universal health coverage and to save lives. The Global Conference on Primary Health Care 2018: from Alma-Ata towards achieving Universal Health Coverage and the Sustainable Development Goals at Astana, Kazakhstan provided a platform for low-and middle-income countries to join the Primary Health Care Performance Initiative (PHCPI). At this Global Conference, Malaysia has declared to become a Trailblazer Country in the PHCPI and pledged to monitor her Vital Signs Profiles (VSP). However, the VSP project requires an honest and transparent data collection and monitoring of the Primary Health Caresystem, so as to identify gaps and guide policy in support of Primary Health Care reform. This is a huge commitment and can only be materialised if there is a collaborative partnership between Primary Care and Public Health providers. Fundamental to all of these, is the controversy concerning whether or not 'Primary Care' and 'Primary Health Care' represent the same entity. Confusion also occurs with regards to the role of 'Primary Care' and 'Public Health' providers in the Malaysian Primary Health Care system. This review aims to differentiate between Primary Care, Primary Health Care and Public Health, describe the relationships between the three entities and redefine the role of Primary Care and Public Health in the PHCPI-VSP in order to transform the Malaysian Primary Health Care system.
\end{abstract}

Keyword : Primary health care; Primary care; Public health; Primary health care performance initiative; Vital signs profile; Trailblazer country; Primary health care reform; Malaysia 